\title{
LOS NUEVOS PARADIGMAS DE LA ADMINISTRACIÓN EN LA UNIVERSIDAD: GLOBALIZACIÓN Y COMPETITIVIDAD
}

\author{
THE NEW PARADIGMS OF MANAGEMENT AT THE UNIVERSITY: GLOBALIZATION AND \\ COMPETITIVENESS
}

Teresa Ríos Delgado*

triosd@unmsm.edu.pe

[RECEPCIÓN: AGOSTO 2015 / CONFORMIDAD: NOVIEMBRE 2015]

\begin{abstract}
RESUMEN
La presente investigación relaciona dos paradigmas actuales de la administración moderna como son la globalización y la competitividad. Los constantes cambios y avances vertiginosos de la ciencia y la tecnología exigen a la universidad una relación horizontal con la sociedad afianzando las competencias docentes y de investigación, tratando de esta manera de adaptarse a los cambios políticos, económicos y sociales del entorno, convirtiéndose en unidades estratégicas al servicio de la sociedad.

Esta investigación es aplicada y explicativa ya que buscamos conocer los factores causales que explican nuestro problema de investigación. Se utilizó el método hipotético deductivo y las técnicas de investigación fueron la entrevista a profundidad, además de las técnicas de observación y documental. Se pudo determinar que en las políticas públicas no se está viendo el futuro dentro del nuevo paradigma de globalización y competitividad, ya que no existe articulación entre la infraestructura y los programas para el desarrollo de la gestión del conocimiento como motor de desarrollo del país.
\end{abstract}

Palabras clave: Globalización, competitividad, paradigmas de la administración.

\section{ABSTRACT}

This research links two existing paradigms of modern management such as globalization and competitiveness. Constant changes and rapid advances in science and technology require that university to build a horizontal relationship with society strengthening teaching skills and research trying to adapt to political, economic and social environmental changes becoming strategic unit to society

* Doctora en educación, Doctora en gestión y desarrollo. Magíster en educación con mención en docencia a nivel superior, Traductora e intérprete, Abogada. Docente auxiliar e investigadora del Instituto de Investigaciones de la Facultad de Ciencias Administrativas (FCA) de la Universidad Nacional Mayor de San Marcos (UNMSM). Docente de pre y posgrado en Investigación. Coordinadora académica de la Unidad de Posgrado FCA-UNMSM 
The university facing this new scenario has to face a world increasingly ruled by uncertainty and must adapt rapidly to the changes tied to economic, political, social transformations that are becoming faster. It is an applied and explanatory research since we seek to know the causal factors that explain our research problem. The hypothetical deductive method was used and as research techniques used were depth interviews and observation and documentary ones. It has been determined that public policy-related to higher education- is not taking in consideration globalization and competitiveness; without a joint of the infrastructure and the programs for the development of knowledge management as engine of the development of the country.

Keywords: Globalization, competitiveness, administration of paradigms.

\section{INTRODUCCIÓN}

Las organizaciones actuales se enfrentan a nuevos paradigmas de la administración moderna como son la globalización y la competitividad. En este nuevo escenario las organizaciones tienen que enfrentarse a un mundo donde impera la incertidumbre, siendo necesario adaptarse rápidamente a los cambios ligados a transformaciones económicas, políticas, sociales que cada vez son más rápidos. Para ello las organizaciones deben tener una visión clara de las implicancias del fenómeno de la globalización que indica que en el mundo cada vez existe un mayor grado de interdependencia entre las diferentes regiones $\mathrm{y}$ naciones en el mundo, particularmente en lo referente a las relaciones económicas, financieras, políticas, sociales y de comunicación.

Por otro lado, el factor de la ventaja competitiva de las naciones que fue abordado por Michel Porter (2008) implica que cada vez los países que van a la vanguardia del desarrollo económico se basan en los factores internos de cada organización para ser más productivos y por el grado de intervención del Estado y sus políticas que juegan un papel determinante en la competitividad del país. Por ello es importante ver cómo la Universidad, a través de sus propuestas educativas de los posgrados, relaciona entre sus participantes este nuevo paradigma de la administración moderna. Por lo tanto, la investigación se sitúa en la relación de la globalización y la competitividad en las nuevas organizaciones.

Se tiene por ejemplo a Valda (2011) quien describe que las características de los actuales escenarios que involucran transformaciones, cambios y oportunidades, muestran a muchas empresas exitosas que han conquistado mercados y que satisfacen plenamente a sus consumidores, apoyadas de una gerencia, que se ha actualizado e identificado con ellos, dando paso a nuevos paradigmas que la ciencia administrativa ha propiciado en las funciones operativas de las organizaciones en su estructura, liderazgo, y cultura organizacional. Las empresas no pueden seguir bajo una gerencia que actúe de acuerdo a los conocimientos tradicionales, cuando mucho de éstos no está adaptados a las exigencias competitivas del presente. Se necesita dar paso a nuevos paradigmas, aportando conocimientos que se identifiquen con los retos a enfrentar y que se susciten los cambios que le ayuden a obtener resultados beneficiosos.

También Tünnermann y De Souza (2003) en su trabajo nos indican que una de las características de la sociedad contemporánea es el papel central del conocimiento en los procesos productivos, al punto que el calificativo más frecuente que suele dársele es el de sociedad del conocimiento. Asistimos a la emergencia de un nuevo paradigma económico-productivo en el cual el factor más importante no es ya la disponibilidad de capital, mano de obra, materias primas o energía, sino el uso intensivo del conocimiento y la información. Será, entonces, preciso educar para el cambio y la incertidumbre. "La comunidad académica, ha escrito Federico Mayor, deberá tener el coraje de decirle a la juventud que las prerrogativas y certezas ya no forman parte del presente: es en la incertidumbre donde está la esperanza al filo de las sombras y las luces".

Los sistemas de educación superior, dice la Declaración Mundial (París, octubre de 1998) deberían aumentar su capacidad para vivir en medio de la incertidumbre, para transformarse y provocar el cambio. La incertidumbre no debe conducirnos a la perplejidad sino a la disposición para el cambio, a la ampliación y renovación incesante del conocimiento. 
Es así que se justifica este estudio en base a este nuevo escenario mundial donde el nuevo paradigma administrativo que es la globalización y la competitividad que va generando en todas las organizaciones un sentido de incertidumbre para la cual deben cada vez adaptarse a los cambios cada vez más rápidos.

Las nuevas condiciones cambiantes y competitivas en las que se desenvuelven las organizaciones en el mundo moderno hace necesario que personas posean una actitud investigadora que les permita conocer el ambiente de su organización y generar nuevas formas de competir, buscado nuevos productos o servicios para satisfacer las necesidades de sus clientes.

Es por ello que los estudiantes de posgrado deben estar conscientes que en este nuevo paradigma, las organizaciones deben estar preparadas para convertirse en organizaciones competitivas y globales justificando así el presente estudio.

Se entiende que una empresa es competitiva cuando su ambiente de funcionamiento conduce al crecimiento sostenido de la productividad, mejorando sus niveles de competitividad y por consiguiente, aumentando sus ingresos.

Una empresa es competitiva si consigue crear un entorno laboral y de negocios adecuados en los que la producción, la tecnología y las estrategias organizacionales le permitan obtener resultados positivos en el mercado al realizar negocios similares a los de sus competidores.

Las organizaciones ya no se encuentran limitadas por fronteras nacionales ni regionales por ello se han vuelto globales, se interactúa con personas de diferentes culturas, existen mayores transferencias hacia el extranjero, los puestos de trabajo tienden a desplazarse de las naciones desarrolladas a países donde los costos de mano de obra son más baratos. Para ello se necesita de organizaciones que adopten técnicas avanzadas de administración que les permitan no solo su óptimo desarrollo sino, fundamentalmente, la realización humana integral de las personas que las componen y de la sociedad de la cual forman parte.

\section{MÉTODOS}

Esta investigación basa su estudio en la relación existente entre la globalización y competitividad. Es empírica porque es un hecho que ocurre en la sociedad, y aplicada porque se va a enfrentar el problema proponiendo alternativas de solución.

El nivel de la investigación es explicativo ya que buscamos conocer los factores causales que explican nuestro problema de investigación. El diseño básico de esta investigación es no experimental-transeccional-correlacional. Es no experimental debido a que no se harán pruebas; es decir, en esta investigación las variables no van a ser manipuladas. Es transeccional debido a que nuestro objeto de estudio va a ser analizado en un periodo determinado de tiempo. Es correlacional porque se trata de encontrar evidencias de cómo la variable independiente globalización se relaciona con la competitividad.

El método de investigación es el método hipotético deductivo y las técnicas de investigación fueron la entrevista a profundidad e igualmente las técnicas de observación y documental; siendo la unidad de análisis el estudiante y docente de posgrado en las facultades de administración, contabilidad y economía. El muestreo fue opinático o criterial ya que dependía mucho de la disponibilidad del maestrista y/o doctorando o del docente, lo que conllevó a un trabajo de campo bastante difícil y, tal como se señaló líneas arriba, a veces infructuoso.

\section{RESULTADOS Y DISCUSIÓN}

Los aspectos relevantes que se han desarrollado en la presente investigación permiten determinar que en las políticas públicas y algunos docentes universitarios no están viendo el futuro dentro del nuevo paradigma de globalización y competitividad. Consecuentemente, no se está articulando la infraestructura y los programas para el desarrollo de la gestión del conocimiento como motor del desarrollo del país, y no estamos viendo qué nuevas competencias esenciales hará falta crear, qué alianzas estratégicas deberíamos promover, qué nuevos conceptos de servicios educativos deberíamos promover y qué programas de desarrollo deberíamos proteger y crear, para ello se necesita instituciones y organizaciones que adopten técnicas avanzadas de investigación para la generación de conocimiento.

Por otro lado, no se está desarrollando una concientización entre los docentes y alumnos de posgrado sobre los factores de la competitividad y de la globalización en este nuevo milenio. 
Algunos programas de estudios no promueven la generación de la gestión del conocimiento como agente importante para la generación de competitividad. Por lo tanto, seguimos actuando como si lo urgente excluyese lo importante, y consecuentemente el futuro apenas se está explorando.

1. Propuestas de la Universidad en los posgrados.

Las propuestas de las universidades en general son:

- Disponer de una adecuada infraestructura y gestión del talento humano para el desarrollo de la gestión del conocimiento. Esto se torna crítico para el futuro de la nación, lo que nos condenaría a permanecer estacionados en el pasado dejando pasar a la modernidad, por ello es importante el desarrollo de la investigación científica en todas las áreas y el desarrollo de proyectos interdisciplinarios.

- Articular con exactitud el concepto de globalización y competitividad con sus oportunidades y riesgos para el desarrollo de nuestras capacidades y habilidades. Aun muchos docentes y directivos de las universidades no han asimilado el verdadero impacto de la globalización y la competitividad en la gestión del conocimiento, hecho que nos pone en una posición de rezago a comparación de los países desarrollados que sí disponen de políticas, estrategias y planes de desarrollo para incorporar personal con capacidades, además de contar con instituciones para dar el salto cuantitativo en el desarrollo de la gestión del conocimiento.

- Desarrollar alianzas estratégicas con las PYME, que son las empresas más emprendedoras y exitosas de nuestro mercado. Tenemos conocimiento de diversas PYME que en base a esfuerzo han salido exitosas en algunos rubros de la economía y que son motivo de estudio interdisciplinario para extraer modelos de desarrollo y crecimiento innovadores de acorde con todo el bagaje cultural en que se desarrollan, como los procesos productivos, los nuevos management y el desarrollo de su cultura organizacional. A partir de ellas se pueden desarrollar modelos organizacionales para luego crear simulaciones y juegos de negocios con las características propias de nuestra realidad y de los mercados internos y externos.

- Las investigaciones se realizan en forma individual y enmarcadas en la verticalidad de cada rama de la ciencia, Seguimos con los paradigmas de que las ciencias se desarrollan en compartimientos aislados, y no proponemos investigaciones aplicadas en las que se involucren en forma horizontal a las ciencias que no permitiría, administrar mejor nuestros recursos humanos, materiales y financieros, buscar el trabajo en equipos multidisciplinarios que nos permita crear la sinergia innovadora que requieren nuestros proyectos

2. Retos de la Universidad en los posgrados en el nuevo paradigma de globalización y competitividad.

Como hemos visto anteriormente el gran motor de desarrollo científico y tecnológico de los países desarrollados es la creación de mayor conocimiento con un importante apoyo financiero.

Los retos de la universidad en los posgrados son:

- Fortalecer el desarrollo de las pequeñas y medianas empresas con herramientas que les permitan integrarlas al círculo virtuoso de la creación de valor a través del desarrollo de la gestión del conocimiento y la innovación, gracias a una cultura de aprendizaje constante en todos sus procesos productivos y gerenciales.

- Fortalecer el management en los pequeños y medianos empresarios para desarrollar una visión global y de competitividad de las empresas.

- Fortalecer la visión de país competitivo en forma integral, fortaleciendo una cultura de empresarios competitivos y globales a través de alianzas estratégicas con las PYME.

- Fomentar la investigación interdisciplinaria, enfocándose en el diagnóstico del problema, la solución de los problemas, la aplicación de las soluciones y su divulgación a la comunidad académica. 


\section{CONCLUSIONES}

1. Se ha demostrado que existe una relación directa entre la globalización y la competitividad como nuevos paradigmas en la universidad que están generando conciencia de que el camino para administrar la incertidumbre es la generación de la gestión del conocimiento a través de la investigación científica.

2. También es importante resaltar que todas las organizaciones, docentes y alumnos se encuentran involucrados en los procesos de gestión del conocimiento, lo que nos permitirá desarrollar las ventajas competitivas a partir del conocimiento de las oportunidades y riesgos que estás involucran.

3. Es importante incluir a las PYMEs como parte importante en la generación del conocimiento a través de las alianzas estratégicas con las universidades, que nos permitirán desarrollar modelos de gestión exitosos en nuestro medio.

\section{REFERENCIAS BIBLIOGRÁFICAS}

Ander-Egg, E. (2001). Métodos y técnicas de investigación social - Acerca del conocimiento y del pensar científico. Buenos Aires: Editorial y distribuidora Lumen SRL.

Anzola, M. O. (2003). Una mirada de la cultura corporativa. Editorial. Universidad externado de Colombia. Colombia.

Blanco Prieto A. (2007). Trabajadores competentes: Introducción y reflexiones sobre la gestión de recursos humanos por competencias. Madrid: ESIC.

Bruckmann, M. (23 de agosto de 2012). Instituto de Pesquisa Económica Aplicada-IPEA de Brasil. Obtenido de http://www.bcv.org.ve/ jornadas/documentos/seminarioMonicaBruckman2012.pdf

Brunet, L. (1999). "El Clima de Trabajo en las Organizaciones" En: Definiciones, diagnóstico y consecuencias. México D.F.: Editorial Trillas.

Consejo Nacional de competitividad (15 de Agosto de 2015). Obtenido de http://www.cnc.gob. pe/web/inicio.php

Dessler G., Varela R. (2004). Administración de Recursos Humanos. Enfoque Latinoamericano. $2^{\mathrm{a}}$ Ed. México D.F.: Pearson Education de México.
Diario El Peruano, Ley 28015, Ley de Promoción y Formalización de la MYPE, promulgada el 3 de julio del 2003.

Diario El Peruano, Decreto Legislativo $N^{\circ} 1086$, Ley de Promoción de la Competitividad, Formalización y Desarrollo de la MYPE y del acceso al empleo decente publicado el 28.06.2008.

Diaz, J., \& Moulines, U. (2008). Fundamentos de la filosofia de la ciencia. Barcelona: Editorial Ariel.

Fairlie Reinoso, A. (2005). Las PYME industriales Exportadoras: El Caso de la región Norte. Lima - Consorcio de Investigación Económica y Social.

Furnham A. (2008). Personality and intelligence at work: exploring and explaining individual differences at work. Psychology Press: East Sussex.

Khun, T. (2002). La estructura de las revoluciones. Barcelona: PAIDOS.

Khun, T. (2000). El camino de la estructura. Barcelona: PAIDOS.

Koontz, H. Welhrich, H. (2004). Administración: Una Perspectiva Global. Editorial Mc Graw Hill. $12^{\mathrm{a}}$ edición. México.

Maristany J. (2007). Administración de Recursos Humanos. $2^{\text {a }}$ Ed. México D.F.: Pearson Education de México.

Medina, G. (2010). Analisis comparado de factores de empresariealidad y competitividad. Lima: Universidad Nacional Mayor de San Marcos.

Méndez Álvarez, C. (2006). Clima organizacional en Colombia, Bogotá. El IMCOC: Un método de análisis para su intervención Centro Editorial Universidad del Rosario.

MINISTERIO DE LA PRODUCCIÓN, (2015). Estadísticas de la Dirección General de MYPE y Cooperativas.

Münch, L. (2005). Métodos y Técnicas de Investigación. $2^{\mathrm{a}}$ Edición. México D.F.: Editorial Trillas.

Porter, Michael (2008). Ventaja competitiva de las naciones. Décimo quinta edición. México D.F.: Compañía Editorial Continental S.A. 
Porter, M. (2009). Estrategia Competitiva: Tecnicas para el analisis de la empresa y sus competidores. México D.F.: Piramide.

Porter, M. (2008). Ventaja Competitiva. México D.F.: Compañia editorial Continental SA de $\mathrm{CV}$.

Porter, M. (2012). Ser competitivo. España: Deusto Grupo Planeta.
Tünnerrnann Bernheim, Carlos y De Souza Chaui, Marilena. 2003. Desafíos de la universidad en la sociedad del conocimiento, cinco años después de la conferencia mundial sobre educación superior. Comité Científico Regional para América Latina y el Caribe del Foro de la UNESCO. París.

Valda, JC. (2011). Grandes PYMES, http://www. pysnnoticias.com/2010/12/28/nuevosparadigmas-en-la-gerencia-moderna/. 